\title{
Performance Appraisal Effect on Compensation
}

\author{
Jacques Touma \\ Lebanese American University, Beirut, Lebanon \\ Email: jacques.touma@lau.edu.lb
}

How to cite this paper: Touma, J. (2022). Performance Appraisal Effect on Compensation. Journal of Human Resource and Sustainability Studies, 10, 1-12.

https://doi.org/10.4236/jhrss.2022.101001

Received: January 2, 2022

Accepted: February 18, 2022

Published: February 21, 2022

Copyright (c) 2022 by author(s) and Scientific Research Publishing Inc. This work is licensed under the Creative Commons Attribution International License (CC BY 4.0).

http://creativecommons.org/licenses/by/4.0/ (c) (i) Open Access

\begin{abstract}
Human Resources function has been evolving throughout the years to reach more effectiveness in the corporate world. Today human resources focus on five major components: recruitment and selection, training and development, pay and benefit, performance appraisal and labor relation. It is worthwhile to mention that Human Resources department works best in a dynamic economy. This article tackles the performance appraisal part and its effectiveness when it comes to compensation. It starts by focusing on the importance of having a clear job description, evaluating employees according to job description using effective job evaluation method and performance appraisal selective methods according to the structure and the field of the company. Linking these tools to different ways of compensating employees and the effectiveness of such actions, problems, diagnostic approach and recommendation of Performance Appraisal (PA) process are covered in this study.
\end{abstract}

\section{Keywords}

Performance Appraisal (PA), Key Performance Indicators (KPIs), Compensation, Effective Performance

\section{Introduction}

The performance appraisal reflects its benefits on individuals and organizations. Its purpose is the maximization of the individual performance as well as the organizational performance thus enhancing the organizational profitability.

Appraisal offers a valuable opportunity to focus on work activities and goals, to identify and correct existing problems, and to encourage better future performance. The retrospection part of the appraisal evaluates the achievement and performance of an employee, so gaps can be identified. The effect of performance appraisal covers three main streams:

- Motivation and satisfaction of the employee: The recognition of an em- 
ployee's effort is a powerful incentive. Thus adjustment of employee's behavior and enhancement of employee's performance can be reached accordingly.

- Training and Development: Performance appraisal offers an excellent opportunity for a supervisor and subordinate to recognize and agree upon individual training and development needs. These needs will be linked to performance outcomes and future career aspirations.

- Recruitment: Appraisal data can be used to monitor the success of the organization's recruitment practices.

The process of compensation once performance appraisal system is in place along with a systematic approach of job evaluation can proceed with its different components.

Performance management process depends on the leaders within the organization and depends on the influences exercised whether strong or weak that can lead to performance or no performance by the respondents (Hawke, 2021).

Therefore, the importance of tackling the right and systematic adaptation of performance appraisal is essential and must be part of every organization as discussed in the findings.

\section{Performance Appraisal}

\subsection{Definition and More}

System evaluation to ease the communication between both the managers and employees that leads to continuous periodic feedback is essential for employees to improve their performance at work and at the same time the employees would feel they are involved not only in their daily practice by performance relates as well to the main objectives of the company. Therefore structured feedback and improvement on the areas of weaknesses and compensation when exceeding performance expectation while taking in consideration transparency and fairness can lead to a great performance system (Werber et al., 2012).

Performance appraisal is a structured formal interaction between a subordinate and supervisor. It usually takes the form of a periodic interview, in which the work performance of the subordinate is assessed to provide recognition for the work efforts and to identify weaknesses and strengths as well as opportunities for improvement, performance maximization, skills development and career perspective.

When these conditions exist, employees will view the appraisal process as accurate and fair. They will also express more acceptance of the appraiser's feedback and a greater willingness to change.

The performance appraisal reflects its benefits on an individual and organizational basis. Its purpose is the maximization of the individual performance as well as the organizational performance thus enhancing the organizational profitability.

Appraisal offers a valuable opportunity to focus on work activities and goals, to identify and correct existing problems, and to encourage better future per- 
formance. The retrospection part of the appraisal evaluates the achievement and performance of an employee, so gaps can be identified. The effect of performance appraisal covers three main streams:

- Motivation and satisfaction of the employee: The recognition of an employee's effort is a powerful incentive. Thus adjustment of employee's behavior and enhancement of employee's performance can be reached accordingly.

- Training and Development: Performance appraisal offers an excellent opportunity for a supervisor and subordinate to recognize and agree upon individual training and development needs. These needs will be linked to performance outcomes and future career aspirations.

- Recruitment: Appraisal data can be used to monitor the success of the organization's recruitment practices.

The process of compensation once performance appraisal system is in place along with a systematic approach of job evaluation can proceed with its different components (Rao, 1985).

Today as Human resources evolve, it is crucial for managers to guide, motivate and lead employees on a daily basis. These organizations have found that the best way to increase employees' motivation and productivity is by having an effective appraisal system.

In general, the performance appraisals are done so the employee would be able to know the performance needed to meet the supervisor's expectations and in case weaknesses are revealed, training sessions should be presented. According to Fletcher (2008), "the evaluation is the starting point of any appraisal system", so the organization will have the opportunity to provide training to employees to improve related performance. Furthermore, the organization will be able to collect records about employees' capabilities and characteristics which can be used for developmental and planning needs at a later stage.

As much as it's beneficial to conduct Performance Appraisal for the company, it is for employees as well. A well designed and effective Performance Appraisal helps employees as follows (Rao, 1985):

- To know more about their roles and functions inside the organization.

- To understand their strengths and weaknesses.

- To see if they are involved and satisfied inside the organization.

- To increase communication with their supervisors and understand their expectations.

- To move to higher positions in the future by reinforcing their characteristics and developing them.

- To enjoy giving their best inside the organization by focusing more on their goals and getting the desired rewards.

Performance management system is widely used today for Performance Appraisal that has many advantages such as reducing paperwork, linking pay to performance through formulation that can be measured by the end of the performance period, infrastructure for the data throughout the organization which 
is a great book keeping from one end and a database for continuous updating from another end.

The performance management system has macro objectives such as retaining top performers and identifying poor performers, simplifying the process of restructuring the assignments to employees and gives the supervisors the ability to manage their subordinates through key performance indicators measurement (Levine, 2017).

The goal of performance appraisal is to evaluate the work results of the employees and shed the light on whether certain tasks were under or over delivered. It is used for the purpose to link that performance to compensation which is in fact increase the motivation of the employee and fulfill the intrinsic need (Mohammed, 2020).

\subsection{Effective Performance Appraisal}

Performance appraisals have positive impacts on both organizations and employees. In order to be effective, these appraisals should be reliable and consistent. They should concentrate on the environment, the employee, and the employee's job. Any subjectivity and traits not related to the job should be excluded from the appraisal. To be effective, it should have "a clear mission, a good system, effective managers and motivated employees all operating together to get the job done" (Logenecker \& Fink, 1999). Therefore, communication is an essential element for any appraisal system and can be downward going from upper management to lower levels as well as upward allowing the employees to communicate their needs and goals. Lack of such communication especially when employee is being appraised may be considered as approval of current performance which can be a negative point towards the employee in an organization. However, the manager can use the legitimate power to interfere in all performance levels to ensure more improvement and satisfactory performance level for both organization and employees (Boice \& Keliner, 1997).

\subsection{Different Components of Performance Appraisal}

Depending on the nature of the company whether it is a service provider, distribution, production and manufacturing, the selection of the ultimate method should be identified and used accordingly. For instance in a production and manufacturing setting there should be multiple evaluations. For the blue collar worker or non-exempt employees so to speak, the usage of work standards is the right selection simply because it relies on production rates for a normal worker at a normal pace. Time and motion studies are performed to establish line balancing and simulation analysis leading to set production and engineering rates for the different manufacturing processes. Therefore, objectives are identified to the worker according to the standards established by management.

As for the while collar and professional staff, there would be a different method to use such as the result-based systems for Engineers within that company 
because it shows how well results are accomplished but lacks employee development therefore it should be coupled with rating scales to be used also for administration staff since it is easy, quick, uses predefined factors on a scale level.

Moreover, Performance appraisal is a formal event occurring normally once or twice a year which states clear criteria that can be used in the evaluation process. It is a quantitative judgment of the employees' performance in which results should be shared (DeNisi \& Pritchard, 2006). Therefore, different techniques can be used by the organization to evaluate their employees such as the ranking method, narrative method, 360 degree method and so forth.

The performance should follow sequential actions to be performed at best possible ways. For instance it involves many layers within the organization and it starts by having the benefit and administration (B\&A) officer in the human resources department send a reminder to the manager to start the appraisal process. In case this is done annually, the date should be in the beginning of the first fourth quarter of the year. Along with the reminder the B\&A officer sends also an updated complete file of the employee to ease out the whole process. The next step is for the manager to conduct the appraisal and give feedback then return complete file back to human resources. At that point the B\&A officer analyze the information and deliver an output of the performance level statistical chart to the human resources manager (HRM) and inform the general manager (GM) of the company or business unit. The last step for the B\&A officer is to calculate the annual bonus of the employee being assessed and sends a copy to both HRM and GM. A copy of potential training(s) to employee should also be sent to training and development (T\&D) specialist. At that point approval should be released from HRM while informing the GM of such action and release of decision goes to payroll for proper pay adjustment (Illustration of this operation is presented in Figure 1 (below).

There are many other means to evaluate employees and it all depends on the type of the company, management style and the industry as well. Ranking method is used in some companies where the employee is evaluated in order (top down). This method is best to use when managers have to make evaluative decisions such as who is getting the raise, promotion, and the employee of the month. In addition, this method can show the employee's ranking in the organization by simple comparison among the rest of the staff. In another setting, narrative method is used or so called "Essay" which is considered more subjective where managers write small essays describing weaknesses, strengths, and behaviors of the employees. This method is more used with executives and professionals with excellent writing ability. One last approach is worthwhile to mention which is the 360 degree appraisal by which the employee's performance will be evaluated by internal and external clients such as managers, customers, colleagues, and even self-evaluation. It is popular yet cost and time consuming approach, where competencies and behaviors will be evaluated. The advantage aspect of this method is the ability for the employee to look at 360 degree evaluation from 


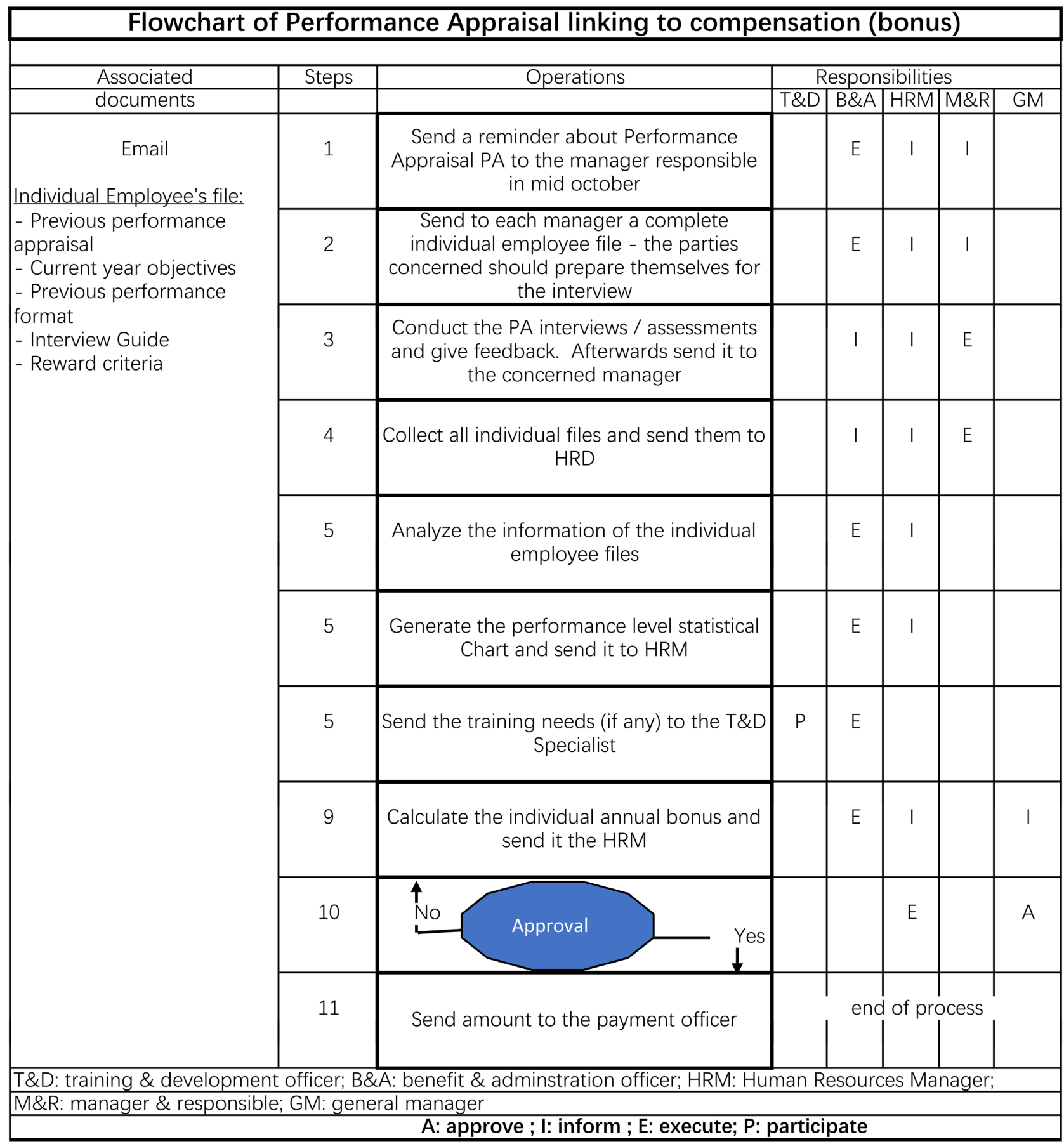

Figure 1. Flowchart of performance appraisal linking to compensation.

peers, supervisors, colleagues and customers therefore identify strengths and weaknesses for further improvement through the T \& D officer and training programs assignment. The other advantage of this method is to reduce discrimination and other types of subjectivity (DeNisi \& Pritchard, 2006).

\subsection{Problems in Performance Appraisal}

The performance appraisal systems tend to have several problems where evalua- 
tions become more controlled by raters' subjectivity and motivational state. Managers can apply different standards for assessments which are unreliable and inconsistent. For example, some employees will be appraised based on recent activities only instead of being appraised during all performance period while others are evaluated based on negative facts which can be noticeable more than positive ones known as "recent behavior bias". Managers can be also "Personal bias" and exercise adverse impact to different employees which directly affect the assessment results. Many other problems in the evaluation could be noticed such as central tendency, leniency, strictness, Halo and Horn error effect, manipulating the evaluation and so forth.

There are multiple ways where appraisals can fail to meet their objectives; however, companies should move quickly to end up with accurate performance methods by developing accurate performance raters, training evaluators, and using multiple raters. Different methods and criteria can be used to evaluate the employees and therefore minimize the chances of inaccuracy of results.

Performance management is affected by many influences such as behavioral, leadership, management and when these influences are weak, there is almost no performance by the respondents according to the study performance by Hawke (2021).

\section{Performance to Compensation}

Compensation is the reward provided to the employees in return for their work. It is the important decisive factor for an organization in order to survive and grow. There are financial and non-financial compensations.

The financial compensation is known also as the extrinsic needs of Maslow, which are the pay that an employee receives as direct compensation such as basic salary, incentives and benefits. Whereas the non-financial compensation known as the intrinsic needs of Maslow, where the employee receives satisfaction from the job itself or from the physical environment in which the job is performed (Chen et al., 1999).

According to Lazear (2018), the pay rate and compensation that the employee receives has a direct impact on the employee's behavior. In another term these incentives and work performance make the economy rolling and consist the overall labor part of GDP.

The organization should maintain, attract and motivate qualified staff in order to do the work effectively. It should work on the benefits such as medical insurance, holidays, kids voucher, education, training and development, membership at a leisure club and so forth (Murray, 2002). This will show the commitment of the organization to the employees and the interest of their well-being. It also opens up channels for communication where employees can express their concerns more openly.

Performance leads to reward and this is a sensitive issue with everyone, if for any reason an employee gets a faulty reward, then the motivation and productiv- 
ity level will decrease and thus the employee feels the effort put at work is not being recognized (Hearn, 2012).

The compensation system should be aligned with the mission, vision and strategy in order to create a compensation plan in an efficient way to achieve the organization goals.

A compensation program in an organization is the result of the establishment of job evaluation such as the point method used in many companies, clear job description and performance appraisal. This is the basic requirement. There are continuous improvement that should be periodically considered in an organizational setting.

\subsection{Continuous Improvement}

Culture audit is a continuous improvement and should be done preferably by third party organizer where organization culture exerts a powerful influence on individual and team performance. The exercise should include steps on how individuals consider the effect of organization culture and identify how to make the soundest possible contribution. The elements of such exercise could include authority, goals and objectives, conflict resolution, commitment, critique, productivity, standard of excellence and so forth. Identifying the soundest measure in each element and comparing result to actual practices in the company is the idea way to discover and audit the culture.

Salary surveys is another continuous improvement for the purpose to identify the correct market price for a position, taking into consideration organization size, location, field of business and the type of business. The top management along with the owners of the company may choose the strategic positioning in terms of the pay level whether to be a pay follower, pay leader, proactive or market rate.

Organization seeking to have a competitive edge has to have a clear salary structure to let employees see how their career can progress in that organization and what rewards may be offered which often leads to decision making based on the interest, feeling and fairness. The salary structure is a guidance for employees in a classification job evaluation corporate set-up to identify ways to increase their salaries within the grade or by moving up the ladder therefore working on increasing the salary and receiving promotions from one grade to another (Anonymous, 2003).

Equity is another mean of affecting the compensation thus promoting the fact that all employees in the organization should be treated equally, and in fact it is the key element in creating successful compensation systems considering the output over input of one colleague is equal to the output over input of another colleague having same job description and qualification. What differs here is the job evaluation used in different organizations that may lead to inequity therefore three options are present to employee whether to standstill, quit or appeal.

For an organization to have an efficient strategy, it should link the perfor- 
mance with rewards because the bottom line will be affected. It is essential for the employee to be satisfied with the pay because it affects the main organizational outcome such as absenteeism, turnover and job satisfaction (Ducharme et al., 2005).

Compensation and rewards are paid to attract qualified employees and motivates them to perform their duties in the most effective and efficient way. The relation between the organization and the employee should be a "win-win" relation, where Human Resources are responsible to motivate people. The Human Resources should communicate a realistic job preview (RJP) and in return explain the benefit package to the employee that enriches the communication and positive labor relation with Human Resources. Compensation and rewards should be legal, equitable, provide security and motivate people to perform the job at maximum efficiency.

\subsection{Components of Compensation}

The components of compensation are based on base pay, performance related pay, fringe benefits, incidental pay and the merit rating.

Base Pay:

The base pay is the time spent on the job which identifies the requirements to fulfill a specific set of duties. It is the pay received for a given period but not including the overtime work. It is a base hourly rate of pay or an annual salary.

\section{Performance-Related Pay:}

The Performance-related pay is a financial reward to the employee whose work reached a required standard, where a specific standard and objective are set, and they should be implanted and applied before a bonus is rewarded. The standard or objective should be related to performance quality and team effectiveness. The employees are familiar with the dos and don'ts at work and encouraged to improve their performance.

Fringe Benefits:

The fringe benefits are the benefits provided in relation to employment and excused from taxation. Some types of fringe benefits could be company car, housing, etc.

Incidental Pay:

The incidental pay is when the employee get quickly appreciated for a job well done and therefore is rewarded by the supervisor. Some examples may be dinner party, lunch, etc. (Chen, Ford, \& Farris, 1999).

\section{Merit Rating:}

Merit rating is when the employee gets a separate bonus or extra step in the job salary scale therefore is rewarded annually when the performance appraisal result is attained. Each organization is supposed to have a salary scale for each position.

The Human Resources should set policies and procedures for rewards and these policies and procedures should be dispersed to the managers in order to 
apply them. If the Human Resources do not write the policies and procedures, it will reflect negative results on the employees (Gohari et al., 2013).

The managers should take advantage of the expectancy theory to motivate and satisfy people and show the relation between their efforts and performance in order to get the reward the employee desires, which differs from one employer to another.

\section{Findings}

In the effort to set your company apart and aim for employees' effectiveness while performing their jobs, there are major tasks to be performed by the human resources department to ensure policies and procedures are well prepared yet implemented, job descriptions and job specifications are clear, job evaluation method is set, performance appraisal is conducted and so forth.

The list of tasks includes but not limited to the below:

- The program should be tailored depending on the firm nature.

- The managers should be trained to interfere in the PA in an effective way and less subjectivity.

- Even if evaluation is done once or twice per year, the employee should be aware of his performance all over the year which request better interference and communication with the managers.

- It is not enough to show the employee that the performance is poor; employees should be given specific instruction to improve for a better performance and developmental issues in the future.

- Targets and expectations should be set and agreed by both managers and employees.

- The appraiser should always concentrate on the employee and should take into consideration his work's nature for a better understanding of the obstacles that might prevent his improvement.

Key Performance Indicators (KPIs) in Human resources functions should be met which basically address directly the motivation, satisfaction, safety (meeting the legal mandate) of the employee but in case of system failure, a diagnostic approach should be considered which states the following:

First step is to diagnose the problem: re-assess the process and perform SWOT analysis, Gap analysis and Cost Benefit analysis to identify why productivity is decreasing or why the rewards are not satisfying the employees.

Second step is to design the solution: in case of dissatisfaction in performance, set a plan B to proceed with a different method of job evaluation, salary structure and so forth.

Third step is to implement the solution: start with a pilot testing implementing plan B to a specific department, the one that has the most dissatisfaction counts for instance. Upon successful implementation switch to full fledge across the board implementation.

Final step is to evaluate the solution: In the next appraisal or culture audit 
evaluate the effect of such implementation on the continuing satisfaction and motivation of the employees.

\section{Conclusion}

The purpose of any appraisal system is to improve both employees and organizations taking into consideration that the employee is the basic resource for the assessment. Any appraisal system chosen by the company should lead to the motivation and satisfaction of the employees where open communications between employees and managers are essentials. Therefore in a competitive market, organizations and managers should ensure that their employees are well involved, motivated therefore committed to the Excellency of the organization with one thing in mind which is reaching both instrumental and terminal values according to Maslow. The employee must feel and believe that the effort of contributing to the firm has a return in value whether it's intrinsic or extrinsic or both to reach personal satisfaction with one aim in mind increasing productivity.

Finally, there are many components to compensation considering the employee is in good status and perceived well by his supervisor and everyone else in the company. Performance Appraisal has a direct effect on compensation as discussed, indicated and illustrated in Figure 1 (below), but the fact is that the financial compensation can meet the lower needs of the employee. In a perfect corporate setting, organizations are looking always to enhance the upper needs of the employees which are the self-esteem and self-actuality with the purpose to sustain them since they represent the greatest assets of the company especially if employees are fully satisfied and motivated which will lead to the ultimate goal of increasing productivity.

This paper addressed Performance Appraisal part of the Human Resource function and its effectiveness. A limiting factor of approaching employees during the pandemic was a common excuse that most of them were overwhelmed not to mention that some were at remote locations and therefore had to use online platform to conduct the research.

\section{Conflicts of Interest}

The author declares no conflicts of interest regarding the publication of this paper.

\section{References}

Anonymous (2003). Compensation Resources. IOMA's Report on Salary Surveys, 3, 8-9.

Boice, D. F., \& Keliner, B. H. (1997). Designing Effective Performance Appraisal Systems. Work Study, 46, 197-201. https://doi.org/10.1108/00438029710367622

Chen, C. C., Ford, C. M., \& Farris, G. F. (1999). Rewards. IEEE Potentials, 18, 10-12. https://doi.org/10.1109/45.755073

DeNisi, A. S., \& Pritchard, R. D. (2006). Performance Appraisal, Performance Management and Improving Individual Performance: A Motivational Framework. Management and Organization Review, 2, 253-277. 
Ducharme, M. J., Singh, P., \& Podolsky, M. (2005). Exploring the Links between Performance Appraisals and Pay Satisfaction. Compensation and Benefits Review, 37, 46-52.

Fletcher, C. (2008). Appraisal, Feedback and Development: Managing Performance Review at Work. Oxford: Routledge.

Gohari, P., Ahmadloo, A., Boroujeni, M., \& Hosseinipour, S. J. (2013). The Relationship between Rewards and Employee Performance. Interdisciplinary Journal of Contemporary Research in Business, 5, 543-570.

Hawke, L. (2021). Performance Management for Success: Public Sector Organizations in Australia and the Philippines. ANU Press.

Hearn, S. (2012). The Crucial Link between Performance and Reward. Strategic HR Review, 11, 39-40. https://doi.org/10.1108/shr.2012.37211aaa.003

Lazear, E. P. (2018). Compensation and Incentives in the Workplace. The Journal of Economic Perspectives, 32, 195-214. https://doi.org/10.1257/jep.32.3.195

Levine, P. (2017). The NSPS Pay-for-Performance System. In Civilian Personnel Reform at the Department of Defense: Lessons from the Failure of the National Security Personnel System (pp. 49-76). Institute for Defense Analyses.

Logenecker, C. O., \& Fink, L. S. (1999).Creating Effective Performance Appraisals. Industrial Management, 41, 18-23.

Mohammed, A. (2020). Review Paper on University Teachers Performance Appraisal. Open Access Library Journal, 7, 1-18.

Murray, S. (2002). Marrying Performance with Reward. Financial Times.

Rao, T. V. (1985). Performance Appraisal Theory and Practice. New Delhi: Vikas Publishing House.

Werber, L., Daugherty, L., Keating, E. G., \& Hoover, M. (2012). Assessment Criterion G: Process for Performance Appraisal Feedback. In An Assessment of the Civilian Acquisition Workforce Personnel Demonstration Project (pp. 41-44). RAND Corporation. 